Short Communication

\title{
Carbon Dioxide Laser Supersonic Drawing Nanofiber Sheet for Extraction of Polycyclic Aromatic Hydrocarbons in Water Samples
}

\author{
Ikuo UETA*1, Naho SEKIGUCHI ${ }^{1}$, Akihiro SUZUKI ${ }^{1}$, Yuta KOBAYASHI ${ }^{1}$, Tetsuo KUWABARA ${ }^{1}$, \\ Yoshihiro SAITO ${ }^{2}$ \\ ${ }^{1}$ Department of Applied Chemistry, University of Yamanashi, 4-3-11 Takeda, Kofu 400-8511, Japan \\ ${ }^{2}$ Department of Applied Chemistry and Life Science, Toyohashi University of Technology, 1-1 Hibarigaoka, Tempaku-cho,
} Toyohashi 441-8580, Japan

\begin{abstract}
An extraction capillary packed with a polyethylene terephthalate nanofiber-sheet was applied for the extraction of five water soluble polycyclic aromatic hydrocarbons (PAHs, including naphthalene (Nap), fluorene (Flu), phenanthrene (Phe), fluoranthene (Flt), and pyrene (Pyr)), in water samples. The extracted PAHs were eluted with acetonitrile, and on-line injected into a high-performance liquid chromatographic system coupled with a fluorescence detector. The limit of detections of the method for Nap, Flu, Phe, Flt, and Pyr were 1.0, 1.0, 0.2, 0.5, and $0.2 \mathrm{ng} / \mathrm{mL}$, respectively, at a sample loading volume of $1.0 \mathrm{~mL}$. The spiked recoveries of the analyte PAHs were in the range of 97.3 to $102 \%$ upon spiking the PAHs into tap water and river water samples.
\end{abstract}

Keywords: Nanofiber sheet; PAHs; Extraction; HPLC

\section{Introduction}

Polycyclic aromatic hydrocarbons (PAHs) are environmental pollutants that are also hazardous to human health [1]. PAHs are generated primarily by incomplete combustion of organic compounds (e.g., oil, coal, and wood). Therefore, PAHs are generally present in atmospheric air [2], and PAHs are also included in soil, sediment, and water [3]. In particular, low molecular weight PAHs are water soluble and they have been detected in several water samples, such as river, sea, and drinking water $[3,4]$.

Gas chromatography (GC) [5-7] and high-performance liquid chromatography (HPLC) [3] have been used for determination of PAHs. Because most PAHs have good fluorescent responses, HPLC coupled with a fluorescence (FL) detector is a promising method for the detection of trace PAHs $[8,9]$.

Liquid-liquid extraction (LLE) have been used for extraction of PAHs from water samples. However, LLE is

*Corresponding author: Ikuo UETA

Tel: +81-55-220-8552; Fax: +81-55-220-8547

E-mail: iueta@yamanashi.ac.jp time consuming and requires large amount of organic solvent. Miniaturized extraction devices are one of the promising sample preparation devices because they enable reducing sample and solvent volumes, and analytical costs $[10,11]$. Our research group has developed miniaturized extraction capillaries for extraction of organic compounds in HPLC analysis $[12,13]$ and ion chromatographic analysis [14]. The extraction capillary was prepared by packing silica gel particles into a stainless steel capillary with a 0.8 $\mathrm{mm}$ i.d. and $1.6 \mathrm{~mm}$ o.d. Because the extraction capillary can be connected to a conventional six-port valve with a polyether ether ketone (PEEK) nut, simultaneous elution, and injection can be achieved by simply passing a small amount of elution solvent. Recently, we developed an extraction capillary that was packed with a polyethylene terephthalate (PET) nanofiber-sheet [15]. The nanofiber was prepared by the carbon dioxide $\left(\mathrm{CO}_{2}\right)$ laser supersonic drawing (CLSD) method, where the nanofiber is produced by irradiation using a $\mathrm{CO}_{2}$ laser on an original PET fiber in

Received: 15 January 2020

Accepted: 10 February 2020

J-STAGE Advance Published: 22 February 2020

DOI: $10.15583 /$ jpchrom.2020.002 
a supersonic jet [16,17]. Therefore, the CLSD-prepared nanofiber does not include the residual solvent, which may be problem for a nanofiber prepared by the electrospinning method. The extraction capillary packed with the nanofiber sheet showed successful extraction of dibutyl phthalate and di(2-ethylhexyl)phthalate in water samples owing to its higher surface area compared with the original fiber. Additionally, the sheet-shaped extraction medium could also contribute to increasing the contact opportunities of the analytes to the nanofiber.

In this study, extraction and HPLC determination of water-soluble PAHs using the extraction capillary packed with the PET nanofiber sheet was investigated.

\section{Experimental}

\subsection{Chemicals}

Naphthalene (Nap), fluorene (Flu), phenanthrene (Phe), fluoranthene (Flt), and pyrene (Pyr) were purchased from Tokyo Chemical Industry Co., Ltd. (Tokyo, Japan). Acetonitrile (>99.9\%) was obtained from Kanto Chemical Co., Inc. (Tokyo, Japan).

\subsection{PET nanofiber-sheet}

The nanofibers were prepared by the CLSD method. First, the as-spun original PET fibers $(100 \mu \mathrm{m}$ in diameter) were irradiated by a $\mathrm{CO}_{2}$ laser $(4 \mathrm{~W})$ in a supersonic jet. The diameter of the prepared nanofiber was approximately 500 $\mathrm{nm}$, and smooth surface was confirmed by scanning electron microscope images. Seven nanofibers were captured on the winding spool rotated at a constant speed, and a $12 \mathrm{~cm}$ wide nanofiber-sheet (thickness ca. $50 \mu \mathrm{m}$ ) was prepared. The details for preparing the nanofiber-sheet are described elsewhere [17].

\subsection{Extraction capillary}

The extraction capillary was prepared by packing the nanofiber-sheet into a stainless steel capillary as illustrated in Fig. 1. (1) An appropriate length of poly(vinylidene fluoride) (PVF) fishing line was inserted into a stainless steel capillary ( $0.8 \mathrm{~mm}$ i.d., $1.6 \mathrm{~mm}$ o.d, $15 \mathrm{~cm}$ length) as the guide fiber; (2) the nanofiber sheet $(1.5 \mathrm{~cm}$ length and $12 \mathrm{~cm}$ wide, approximately $5 \mathrm{mg}$ ) was loosely rolled, and the rolled nanofiber-sheet was inserted into the loop of the guide fiber; (3) the nanofiber-sheet was introduced into the capillary by pulling the guide fiber, and then the guide fiber was removed; (4) the nanofiber-sheet was pushed by a fused silica capillary; (5) the capillary was cut to a length of $5.0 \mathrm{~cm}$. The packing length of the nanofiber-sheet was approximately $2.5-2.6 \mathrm{~cm}$. The void volume of the extraction capillary was $20 \mu \mathrm{L}$.

\subsection{Extraction and elution of PAHs}

Before extraction of the PAHs in the water samples, the

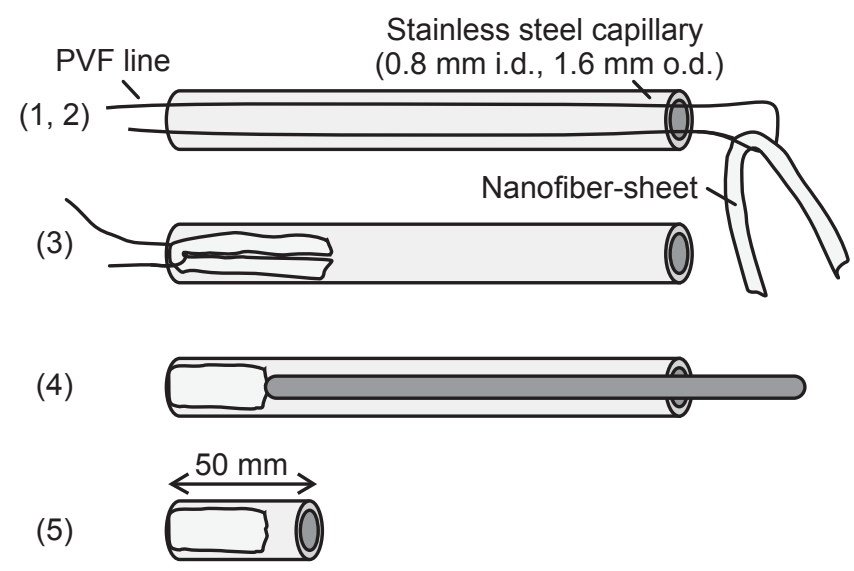

Fig. 1. Illustration of the preparation method for the extraction capillary.

extraction capillary was washed by loading $500 \mu \mathrm{L}$ of acetonitrile, and approximately $500 \mu \mathrm{L}$ of clean air was loaded into the extraction capillary to remove the remaining acetonitrile. The extraction and elution method utilized in this study are illustrated in Fig. 2. The extraction capillary was connected to a Rheodyne model 7725 injection valve (Cotati, CA, USA) with a PEEK nut. Then, a water sample of $100-1000 \mu \mathrm{L}$ was introduced into the extraction capillary by a glass syringe at a flowrate of approximately 1000 $\mu \mathrm{L} /$ min. A further higher sample loading speed was difficult to achieve due to the resistance of the packed nanofibersheet. The extraction capillary can also be connected to the syringe using PTFE tubes. After the sample extraction, the extraction capillary was connected to a six-port valve (Rheodyne 7010) equipped with a $20 \mu \mathrm{L}$ sample loop using a PEEK nut. The other side of the extraction capillary was connected to the Rheodyne injection valve. Desorption of
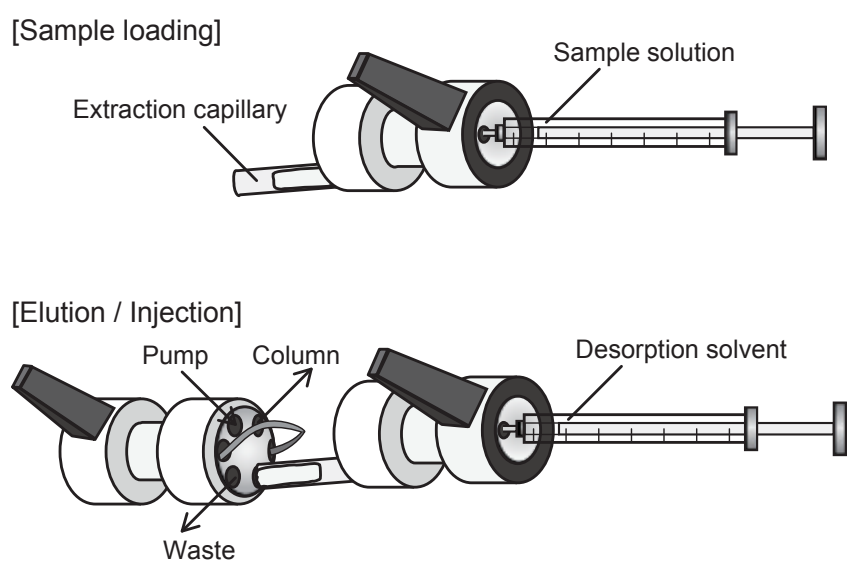

Fig. 2. Illustration of extraction and elution methods using the extraction capillary. 
the analytes was achieved by introducing the desorption solvent into the extraction capillary from the opposite side as where the sample was introduced, as shown in Fig. 2. The desorption solvent $(25 \mu \mathrm{L})$ was first introduced into the extraction capillary to fill the extraction capillary with the desorption solvent, and it was held for $5 \mathrm{~min}$. Then, $20 \mu \mathrm{L}$ of desorption solvent was additionally loaded to fill the sample loop, and injection was performed by switching the six-port valve.

The extraction capillary was washed with $500 \mu \mathrm{L}$ of acetonitrile and dried by introducing air. The extraction capillary could be reused more than 50 times without decreasing the extraction and desorption efficiencies.

\subsection{HPLC measurements}

An HPLC pump (model PU-2080, JASCO, Tokyo, Japan), CO-265 plus column oven (JASCO), and FP-920 fluorescence detector (JASCO) were used for all the chromatographic measurements. The excitation $(\lambda \mathrm{ex})$ and emission ( $\lambda$ em) wavelengths for the analytes are summarized in Table 1. A six-port valve (model 7010, Rheodyne) with $20 \mu \mathrm{L}$ of sample loop was used for sample injection. A ZORBAX Eclipse PAH column (particle size of $5 \mu \mathrm{m}$, internal diameter of $4.6 \mathrm{~mm}$, length of $150 \mathrm{~mm}$, Agilent Technologies, Santa Clara, CA, USA) was employed for the separation. As the mobile phase, $25 \%$ water and $75 \%$ acetonitrile was used at a flow rate of 1 $\mathrm{mL} / \mathrm{min}$. The column temperature was kept at $35^{\circ} \mathrm{C}$. A low-volume pre-column filter $(0.5 \mu \mathrm{m}$ frit, GL Sciences, Tokyo, Japan) was placed in front of the column. A Chromato-PRO data integrator (Run Time Instruments, Tokyo, Japan) was used for data acquisition.

Table 1. Analyte PAHs and their excitation and emission wavelengths.

\begin{tabular}{cccc}
\hline Compound & Abbreviation & $\lambda$ ex $(\mathrm{nm})$ & $\lambda$ em $(\mathrm{nm})$ \\
\hline Naphthalene & Nap & 260 & 330 \\
Fluorene & Flu & 260 & 375 \\
Phenanthrene & Phe & 260 & 375 \\
Fluoranthene & Flt & 260 & 440 \\
Pyrene & Pyr & 260 & 380 \\
\hline
\end{tabular}

\section{Results and discussion}

\subsection{Optimization of the desorption process}

Based on our previous study, desorption of the extracted analytes from the nanofiber sheet was achieved via two-step solvent loadings. Because the volume of the sample loop was $20 \mu \mathrm{L}$, the volume of the desorption solvent used during the 2 nd loading was fixed at $20 \mu \mathrm{L}$. To calculate the desorption efficiency, desorption was performed four times, and each desorption was accomplished with $20 \mu \mathrm{L}$ of desorption solvent. The desorption efficiency was calculated using the peak area of each analyte obtained during the 1 st injection to the total peak areas obtained from the 1st to 4th injections. Acetonitrile and methanol were studied as desorption solvents. The volumes of the desorption solvent for the 1 st loading were 20, 25, and 30 $\mu \mathrm{L}$, where the waiting time between 1 st and 2 nd loadings was fixed at $5 \mathrm{~min}$. The concentration of the analytes was $10 \mathrm{ng} / \mathrm{mL}$, and the sample loading volume was $1000 \mu \mathrm{L}$. The desorption efficiency at a loading volume of $20 \mu \mathrm{L}$ for the 1st solvent loading showed insufficient desorption for all the investigated analytes, where the desorption efficiencies were in the range of 58.9 to $61.7 \%$. Additionally, the peak area obtained at $20 \mu \mathrm{L}$ during the 1st loading was lower than at $25 \mu \mathrm{L}$ and $30 \mu \mathrm{L}$. The highest peak area for all the analytes was obtained at a loading volume of $25 \mu \mathrm{L}$. Therefore, the 1st loading volume for the desorption solvent was determined to be $25 \mu \mathrm{L}$. After the 1st loading, the extraction capillary was held for a few minutes. The hold times were investigated at 0,15 , and $10 \mathrm{~min}$. Because the hold time of $5 \mathrm{~min}$ showed a higher elution efficiency and good repeatability, the hold time was determined to be 5 min. The desorption efficiency using the optimized method of $25 \mu \mathrm{L}$ loading, a $5 \mathrm{~min}$ hold, and $20 \mu \mathrm{L}$ loading using acetonitrile was in range of 71.8 to $84.9 \%$, where the desorption efficiency using methanol was in the range of 44.8 to $67.1 \%$. Therefore, acetonitrile was used as the desorption solvent in this study.

The extraction efficiency for investigated PAHs were also investigated by loading $1000 \mu \mathrm{L}$ of the standard solution, including $500 \mathrm{ng} / \mathrm{mL}$, was introduced into the extraction capillary. The sample solution that passed through the extraction capillary was then analyzed by HPLC, and the extraction ratio was calculated on the basis of a comparison of the peak areas obtained by the extraction capillary passed solution and that by direct injection of the same sample solution. The extraction ratio of high-concentration investigated PAHs were from 93.2 to $94.7 \%$.

\subsection{Method evaluation}

The linearity of the analyte peak area was investigated for sample loading volumes of 100 to $1000 \mu \mathrm{L}$. The concentration of the analyte was fixed at $100 \mathrm{ng} / \mathrm{mL}$. The linearity was also investigated for sample concentration of 5.0 to $1000 \mathrm{ng} / \mathrm{mL}$. The peak area linearly increased in both cases with a correlation coefficient that was above 0.99 for all the investigated PAHs. This result clearly indicating sufficient sample loading capacity of the PET nanofiber-sheet for the investigated PAHs.

The limit of detection (LOD) for the investigated analytes by direct injection (without concentration, $20 \mu \mathrm{L}$ sample loop), and the proposed method (concentration for $1000 \mu \mathrm{L}$ ) are summarized in Table 2. LOD was defined at a 
Table 2. Comparison of LODs for direct injection and extraction.

\begin{tabular}{ccc}
\hline \multirow{2}{*}{ Compound } & \multicolumn{2}{c}{ LOD $(\mathrm{ng} / \mathrm{mL})$} \\
\cline { 2 - 3 } & Direct injection & Extraction \\
\hline Nap & 10 & 1.0 \\
Flu & 20 & 1.0 \\
Phe & 5 & 0.2 \\
Flt & 5 & 0.5 \\
Pyr & 5 & 0.2 \\
\hline
\end{tabular}

signal to noise ratio of 3.3 , and the LOQ was 10 . The proposed method indicated that for $1 / 10$ to $1 / 25$ lower LODs than those with direct injection, the relative standard deviations of the peak areas of Nap, Flu, Phe, Flt, and Pyr at concentrations of $10,10,2.0,5.0$, and $2.0 \mathrm{ng} / \mathrm{mL}$ respectively, were less than $7.8 \%$. Although, typical PAH levels in environmental water samples were normally less than $1 \mathrm{ng} / \mathrm{mL}$ in recent years, the method has satisfactory sensitivity for detection of PAH pollutions in environmental water samples.

Spike recovery of the investigated PAHs using the proposed method was investigated by spiking a standard sample into two river water samples (Kofu, Yamanashi) and a tap water sample (Kofu, Yamanashi). The concentrations of the spiked Nap, Flu, Phe, Flt, and Pyr in the sample solutions were $100,200,50,50$, and $50 \mathrm{ng} / \mathrm{mL}$, respectively. The river samples were centrifuged at $2000 \mathrm{rpm}$ for $10 \mathrm{~min}$ to remove solid matter before measurement. The spike recovery was calculated by comparing the peak areas obtained for the river water sample and standard solution (dissolved in pure water). The analyte PAHs were not detected from the investigated tap water and river water samples. The spike recoveries of the proposed method are shown in Table 3. The results indicated that sufficient recovery of the spiked PAHs was achieved using the proposed method. Figure 3 shows the chromatogram of the analyte PAHs spiked in the river water (A). The PAHs were clearly determined without any significant interference peaks.

Table 3. Spike recoveries of the investigated PAHs.

\begin{tabular}{crrr}
\hline \multirow{3}{*}{ Compound } & \multicolumn{3}{c}{ Recovery (\%) } \\
\cline { 2 - 4 } & $\begin{array}{c}\text { River water } \\
(\mathrm{A})\end{array}$ & $\begin{array}{c}\text { River water } \\
(\mathrm{B})\end{array}$ & Tap water \\
\hline Nap & $97.6 \pm 3.2$ & $100.2 \pm 3.8$ & $101.2 \pm 4.2$ \\
Flu & $97.3 \pm 3.4$ & $98.4 \pm 5.0$ & $100.6 \pm 2.0$ \\
Phe & $101.8 \pm 2.5$ & $100.3 \pm 3.7$ & $100.2 \pm 6.9$ \\
Flt & $97.7 \pm 3.9$ & $100.2 \pm 2.7$ & $102.5 \pm 1.8$ \\
Pyr & $100.9 \pm 4.1$ & $101.1 \pm 1.5$ & $100.4 \pm 3.4$ \\
\hline
\end{tabular}

$n=5$.

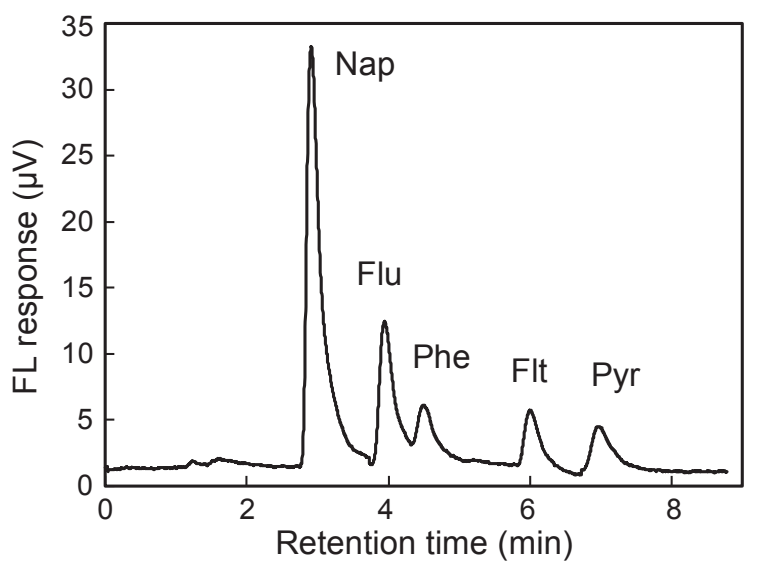

Fig. 3. Chromatogram for the determination of spiked PAHs in the river water sample (A).

\section{Conclusion}

An extraction capillary packed with a PET nanofiber-sheet prepared by the CLSD method was successfully applied for the HPLC determination of water soluble PAHs in water samples. Because the nanofibers had a higher surface area than the original fibers, the extraction capillary showed sufficient sample loading capacity, and linearity for the analytes. Additionally, the nanofibers did not have micropores, and the extracted analytes were easily eluted by passing a small amount of acetonitrile. Owing to the easy preparation of extraction capillary packed with the nanofiber sheet, further application of the device should be considered.

\section{References}

[1] "IARC Monogr. Eval. Carcinog. Risks Hum. Some Non-heterocyclic Polycyclic Aromatic Hydrocarbons and Some Related Exposures", International Agency for Research on Cancer, Lyon, 2010.

[2] "Polycyclic aromatic hydrocarbons" in "WHO Guidelines for Indoor Air Quality: Selected Pollutants" World Health Organization, Copenhagen, 2010.

[3] "Polynuclear aromatic hydrocarbons in Drinking-water" WHO Guidelines for Drinking-water Quality, Geneva, 1998.

[4] Hayakawa, K.; Makino, F.; Yasuma, M.; Yoshida, S.; Chondo, Y.; Toriba, A.; Kameda, T.; Tang, N.; Kunugi, M.; Nakase, H.; Kinoshita, C.; Kawanishi, T.; Zhou, Z.; Qing, W.; Mishukov, V.; Tishchenko, P.; Lobanov, V.B.; Chizhova, T.; Koudryashova, Y. Chem. Pharm. Bull. 2016, 64, 625-631.

[5] Li, A.; Uchimura, T.; Tsukatani, H.; Imasaka, T. Anal. Sci. 2010, 26, 841-846.

[6] Ueta, I.; Onikata, M.; Fujimura, K.; Sasaki, T.; Yoshimura, T.; Mochizuki, S.; Maeda, T. 
Chromatography 2016, 37, 9-13.

[7] Ueta, I.; Onikata, M.; Fujimura, K.; Yoshimura, T.; Narukami, S.; Mochizuki, S.; Sasaki, T.; Maeda, T. J. Sep. Sci. 2016, 39, 4202-4208.

[8] "Method 610: Polynuclear Aromatic Hydrocarbons", United State Environmental Protection Agency, Washington, DC., 1984.

[9] Ueta, I.; Sekiguchi, N.; Fujimura, K.; Yoshimura, T.; Narukami, S.; Mochizuki, S.; Sasaki, T.; Maeda, T. Chromatography 2018, 39, 119-124.

[10] Saito, Y.; Ueta, I. Chromatography 2017, 38, 85-94.

[11] Ueta, I.; Saito, Y. Anal. Sci. 2014, 30, 105-110.

[12] Ueta, I.; Mochizuki, S.; Kawakubo, S.; Kuwabara, T.; Jinno, K.; Saito, Y. Anal. Bioanal. Chem. 2015, 407, 899-905

[13] Ueta, I.; Mochizuki, S.; Kawakubo, S.; Kuwabara, T.; Saito, Y. Anal. Sci. 2015, 31, 99-103.

[14] Ueta, I.; Kajimoto, M.; Saito, Y. Chromatography 2019, 40, 33-37.

[15] Ueta, I.; Sekiguchi, N.; Suzuki, A.; Kobayashi, Y.; Kuwabara, T.; Saito, Y. Anal. Sci. in press. doi: 10.2116/analsci.19P317.

[16] Suzuki, A.; Tanizawa, K. Polymer 2009, 50, 913-921.

[17] Suzuki, A.; Hosoi, K.; Miyagi, K. Polymer 2015, 60, 252-259. 